\title{
Phase behaviour of dehydrated phosphatidylcholines
}

\author{
Samuel K. Owusu-Ware ${ }^{1}$ Babur Z. Chowdhry ${ }^{1} \cdot$ Stephen A. Leharne $^{1} \cdot$ \\ Milan D. Antonijević ${ }^{1}$
}

Received: 10 December 2015/Accepted: 8 November 2016/Published online: 24 November 2016

(C) The Author(s) 2016. This article is published with open access at Springerlink.com

\begin{abstract}
Dehydrated DLPC, DMPC, DPPC and DSPC have been characterised at temperatures below the diacyl carbon chain-melting transition $\left(T_{\mathrm{m}}\right)$, using DSC. For the first time, the existence of pre-Tm transition processes, which are, usually, only observed in the colloidal/liposomal state of saturated phospholipids, has been detected for the dehydrated phosphatidylcholines. Temperature-modulated differential scanning calorimetry was used to characterise the several complexes, overlapping pre- $T_{\mathrm{m}}$ transition processes. Kinetic studies of the chain-melting $\left(T_{\mathrm{m}}\right)$ transition show the activation energy dependence on $\alpha$ (conversion rate), i.e. activation energy decreases as the transition progresses, pointing to the importance of initial cooperative (intra- and inter-molecular) mobility. Furthermore, the activation energy increases with increase in diacyl chain length of the phosphatidylcholines which supports the finding that greater molecular interactions of the polymer chain and its head groups in the dehydrated solid state lead to enhanced stability of dehydrated phosphatidylcholines.
\end{abstract}

Keywords Phospholipids · Dehydrated phosphatidylcholines $\cdot$ DSC $\cdot$ Phase transitions

Electronic supplementary material The online version of this article (doi:10.1007/s10973-016-5957-x) contains supplementary material, which is available to authorized users.

Milan D. Antonijević

M.Antonijevic@greenwich.ac.uk

1 Department of Pharmaceutical, Chemical \& Environmental Sciences, Faculty of Engineering and Science, University of Greenwich (Medway Campus), Chatham Maritime, Kent ME4 4TB, UK

\section{Introduction}

Phospholipids are amphiphilic molecules which consist structurally of diglycerides composed of one glycerol (1,2,3-propanetriol) moiety bonded with two fatty acids at the $s n-1$ and $s n-2$ positions on the glycerol via an ester bond. The third hydroxyl group of glycerol is attached to a phosphoric acid via a phosphate ester bond. Furthermore, the phosphate moiety is bonded with a complex amino alcohol by a second phosphate ester bond. These complex amino alcohols can be choline, ethanolamine or an amino acid, e.g. serine. The functional properties of phospholipids are governed by their ability to undergo lyotropic and thermotropic transitions. Arguably, the most important thermotropic transition in aqueous colloidal suspensions of phosphatidylcholines (PCs) is the gel phase to liquidcrystalline phase which is attributed to the "chain-melting" of the hydrocarbon tail, and the temperature at which this occurs is denoted the $T_{\mathrm{m}}[1,2]$. This transition influences the fluidity of the lamellar structures formed by phospholipids in aqueous media and therefore the stability and the structural integrity of both liposomal formulations and cellular membranes. For saturated phospholipids, it is well documented that the diacyl chain length has a significant influence on the $T_{\mathrm{m}}$ values both in the colloidal state and in the solid anhydrous state [3, 4]. Increasing the carbon chain length of saturated phospholipids results in an increase in the $T_{\mathrm{m}}$, hence an increase in physical stability $[5,6]$.

Previous studies have demonstrated that saturated anhydrous phosphatidylcholines undergo several thermotropic transitions above their $T_{\mathrm{m}}$ values and below the isotropic melts, which occurs at $230{ }^{\circ} \mathrm{C} \quad[7,8]$. Chapman et al. [7] were able to identify the existence of three liquid-crystalline phases at temperatures below the isotropic melt. The thermotropic phase behaviour of the 
phosphatidylcholines has been observed to show similarities in the aqueous colloidal state and in the solid state, differing only by the temperatures at which the processes occur [7]. Therefore, gaining greater understanding of the structural changes/liquid-crystalline transitions in anhydrous phospholipids can shed light on their intrinsic behaviour in living systems and in industrial applications.

The aim of the studies reported herein was to detect the liquid-crystalline transitions and characterise processes leading to the $T_{\mathrm{m}}$ of a homologous series of saturated dehydrated phosphatidylcholines.

\section{Materials and methods}

\section{Materials}

1,2-Dilauryl-sn-glycoro-3-phosphocholine (DLPC), 1,2,dimyristol-sn-glycero-3-phosphocholine (DMPC), 1,2-dipalmitoyl-sn-glycero-3-phosphocholine (DPPC) and 1,2distearoyl-sn-glycero-3-phosphocholine (DSPC) of $\geq 99 \%$ were kindly provided by Lipoid (Germany). The chemical formulae for the phospholipids are $\mathrm{C}_{32} \mathrm{H}_{64} \mathrm{NO}_{8} \mathrm{P}, \mathrm{C}_{36} \mathrm{H}_{72}$ $\mathrm{NO}_{8} \mathrm{P}, \mathrm{C}_{40} \mathrm{H}_{80} \mathrm{NO}_{8} \mathrm{P}$ and $\mathrm{C}_{44} \mathrm{H}_{88} \mathrm{NO}_{8} \mathrm{P}$ for DLPC, DMPC DPPC and DSPC, respectively. Their purity and identity were confirmed by elemental CHN analysis (Table S.1) using a FLASHEA 112 series CHN analyzer (Thermo Electron Corporation). The FT-IR spectra (Fig S.1) acquired using MK11 golden gate (Specac) in the range $4000-600 \mathrm{~cm}^{-1}$ agree with previously reported FT-IR data of the materials [8,9].

\section{Differential scanning calorimetry (DSC)}

All DSC studies were performed using a Q2000 (TA Instruments, UK) calorimeter. A nitrogen atmosphere at a

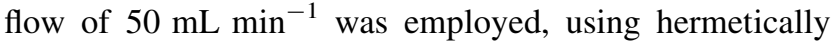
sealed Tzero aluminium pans with a single pin-hole in the lid. Sample mass of $2.5 \pm 0.3 \mathrm{mg}$ and various heating programmes were employed to study the behaviour of each material. Prior to data collection, each sample is initially cooled to $-90{ }^{\circ} \mathrm{C}$ and heated to $140{ }^{\circ} \mathrm{C}$ three times at $10{ }^{\circ} \mathrm{C} \min ^{-1}$. This was to ensure that virtually all moisture in the systems was removed, thereby allowing the behaviour of the dehydrated material to be studied (TG results in Fig S. 2 show moisture content of only $<0.15 \%$ after the phospholipids have been dehydrated by heating to $140{ }^{\circ} \mathrm{C}$ ). The samples were then held isothermal at $-50{ }^{\circ} \mathrm{C}$ for $5 \mathrm{~min}$ and heated to $240{ }^{\circ} \mathrm{C}$ at $10{ }^{\circ} \mathrm{C} \mathrm{min}{ }^{-1}$. Heating rate dependence of the transitions associated with the pre- $T_{\mathrm{m}}$ and the $T_{\mathrm{m}}$ was performed by heating samples from -50 to $125{ }^{\circ} \mathrm{C}$ at $5,10,15$ and $20{ }^{\circ} \mathrm{C} \mathrm{min}{ }^{-1}$. A different sample was used for each heating rate and was subjected to the
-90 to $140{ }^{\circ} \mathrm{C}$ at $10{ }^{\circ} \mathrm{C} \mathrm{min}{ }^{-1}$ heat treatment to remove moisture prior to analysis.

Temperature-modulated DSC (TMDSC) was also performed using sample size of $8.5 \pm 0.4 \mathrm{mg}$. An underlying heating rate of $2{ }^{\circ} \mathrm{C} \min ^{-1}$ with temperature amplitude of $1.5^{\circ} \mathrm{C}$ every $60 \mathrm{~s}$ was employed from -90 to $125^{\circ} \mathrm{C}$. Prior to the temperature modulation, the samples were heated from -90 to 140 three times to remove moisture from the samples.

\section{X-ray powder diffraction (XRPD)}

XRPD analysis of the samples was carried out using a Bruker D8 Advance (Germany) instrument in theta-theta mode. A $\mathrm{Cu}$ anode at $40 \mathrm{kV}$ and $40 \mathrm{Ma}$, parallel beam Goebel mirror, 0.2-mm exit slit, LynxEye position sensitive detector with $3^{\circ}$ opening (LynxIris at $6.5 \mathrm{~mm}$ ) and sample rotation of $15 \mathrm{rpm}$ were used. Each sample was scanned at ambient temperature over the $0.5^{\circ}-40^{\circ} 2 \theta$ range using steps of $0.022 \theta$, counting $30 \mathrm{~s}$ per point.

\section{Results and discussion}

DSC analysis was conducted to gain knowledge of the thermophysical stability of the dehydrated PCs. The results show that all the PCs examined undergo several thermotropic transitions prior to the isotropic melt (at $230{ }^{\circ} \mathrm{C}$ ) (Fig. 1). The temperatures and enthalpies of these transitions are presented in Table 1. DLPC and DMPC undergo an additional transition (8th) at $215 \pm 1$ and $209 \pm 1{ }^{\circ} \mathrm{C}$, respectively. DMPC has another transition at $122 \pm 1{ }^{\circ} \mathrm{C}$ (3rd transition) which was not detected for the other PCs analysed.

It is well documented that when water/solvent is removed, the $T_{\mathrm{m}}$ of phospholipids is raised to higher temperatures. For example, the $T_{\mathrm{m}}$ of DPPC is detected at $41{ }^{\circ} \mathrm{C}$ in liposomes, while the $T_{\mathrm{m}}$ is generally reported at $\sim 100{ }^{\circ} \mathrm{C}$ in the anhydrous state [10]. In this study, $T_{\mathrm{m}}$ values were found at. $91 \pm 1,101 \pm 1,106 \pm 1$ and $109 \pm 1{ }^{\circ} \mathrm{C}$ for DLPC, DMPC, DPPC and DSPC, respectively, which agrees with previously reported values $[7,8,11]$.

For anhydrous phospholipids, the chain-melting transition is attributed to the transformation from a crystalline to a liquid-crystalline phase $\left(L_{\alpha}\right)$ [8]. As such, when the sample is heated above the $T_{\mathrm{m}}$, molecules are arranged into a multi-layered structure. With respect to the highly ordered crystalline form, the liquid-crystalline phase exhibits a greater degree of motional freedom, while it maintains some degree of order, i.e. orientational and/or positional order. 
Fig. 1 Overlay of the DSC data observed for DLPC, DMPC, DPPC and DSPC heated from -50 to $240{ }^{\circ} \mathrm{C}$ at $10{ }^{\circ} \mathrm{C} \mathrm{min}^{-1}$ after removal of moisture from the samples

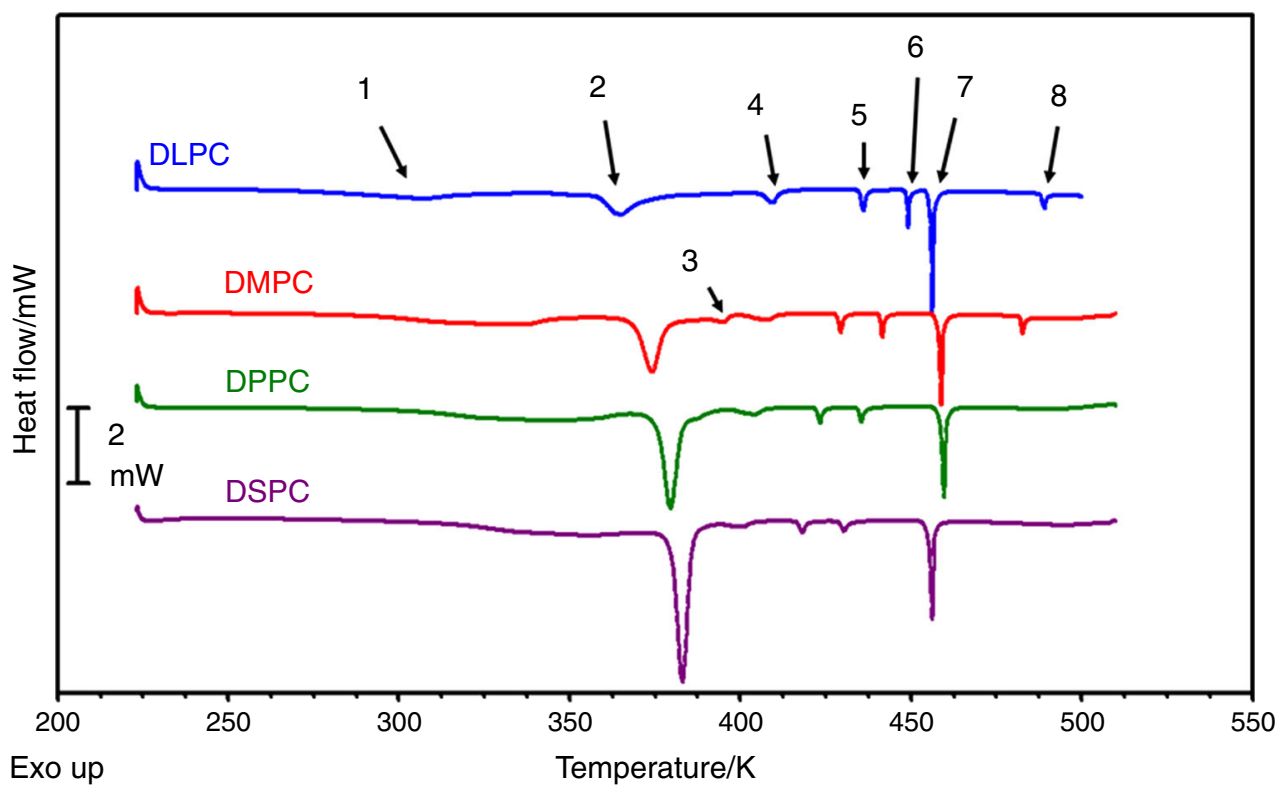

The existence of the broad endothermic process prior to the $T_{\mathrm{m}}$ during a heating scan has been reported only for fully hydrated phospholipids, in the colloidal state, and is usually referred to as the pre- $T_{\mathrm{m}}$ transition [4]. In this study, a pre- $T_{\mathrm{m}}$ transition was observed for all materials studied. This is the first time this process has been reported for the dehydrated phosphatidylcholines in a DSC study.

The characteristics of the pre- $T_{\mathrm{m}}$ transition observed in solution are generally broad and usually associated with a lower enthalpy change, when compared with the chainmelting transition [4]. Furthermore, it has been demonstrated that the temperature difference between this pre- $T_{\mathrm{m}}$ transition process and the chain-melting $\left(T_{\mathrm{m}}\right)$ transition decreases with increasing diacyl chain length. The same behaviour was observed in this study, i.e. the "pre- $T_{\mathrm{m}}$ transition" processes are broad and, as shown in Table 2, the temperature difference between the two processes decreased with increasing diacyl carbon chain length in the dehydrated samples. Therefore, it is inferred that the pre$T_{\mathrm{m}}$ processes observed in this study are similar in behaviour to those observed in the fully hydrated phosphatidylcholines.

The nature of these pre- $T_{\mathrm{m}}$ processes in the anhydrous phosphatidylcholines is very complex. A previous study of DHPC (1,2-di- $n$-heptadecanoyl phosphatidylcholine) suggests that the broad initial endothermic process (the pretransition process) observed during a heating scan is a glass transition, which is then followed by the chain-melting transition [12]. The authors attributed this broad endotherm to the "glassy crystal" or semi-crystalline nature of the material.

XRPD analysis showed the phosphatidylcholines to exhibit very weak diffraction patterns at high $2 \theta$ values
(Fig. 2a, b), which is typical of semi-crystalline materials. It has been reported that the weak high-angle diffractions relate to the chain order for anhydrous phospholipids, i.e. long-range order are observed only at very low $2 \theta$ angles as shown in Fig. 2b [13]. Furthermore, temperature-modulated DSC (TMDSC) results (Fig. 3a-d) showed the samples to undergo transitions that are typical of semicrystalline materials [14-17]. From the TMDSC results, the samples undergo a step-change in baseline of the heat flow signal (R-1 in Fig. 3a-d), which is indicative of a glass transition in the amorphous regions. Overlapping with this signal is a small but broad endothermic process ( $\mathrm{R}-2$ in Fig. 3a-d). Latter transition in the reversing heat flow is not uncommon and has been attributed previously to the partial melting of lamellae of some semi-crystalline polymers $[14,15]$. Hence, the phosphatidylcholines undergo glass transition and partial chain-melting in the dehydrated state, during the pre- $T_{\mathrm{m}}$ process, which may facilitates the progression of the main chain-melting transition at the $T_{\mathrm{m}}$.

Exothermic processes are typically due to crystallisation and commonly appear in the non-reversing heat flow signal of TMDSC results. The reason is that they are usually slow processes and typically out of phase with the modulated temperatures [15]. It is interesting that crystallisation process occurs simultaneously with the chain-melting process (R-3 in Fig. 3a-d). This behaviour may result from some degree of positional and/or orientational ordering - an exothermic process that is fast and most likely to be heating rate dependent - as the material undergoes transformation into the liquid-crystalline phase.

In the non-reversing heat flow signal, each sample undergoes two phase transitions and chain-melting transition (NR-1, NR-2 and NR-3 in Fig. 3a-d, respectively) 


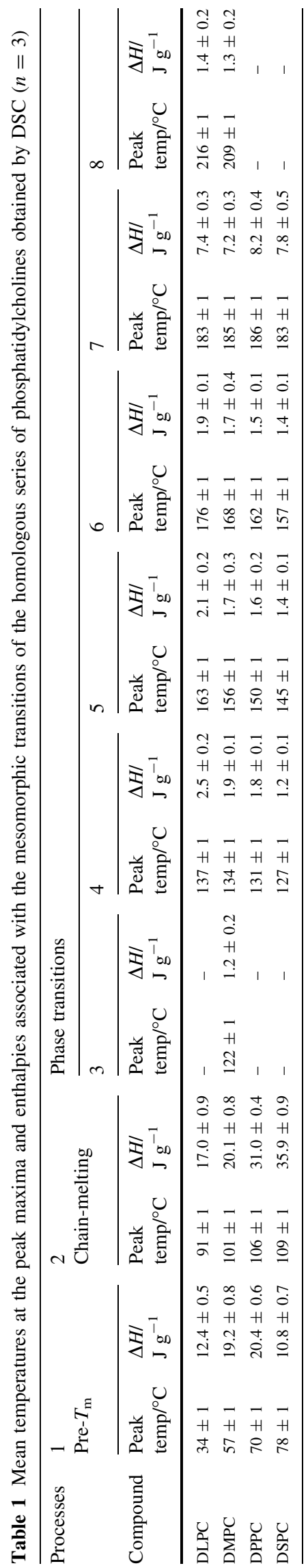

Table 2 Temperatures at the peak maxima of the pre- $T_{\mathrm{m}}$ transition and chain-melting transition processes detected for DLPC, DMPC DPPC and DSPC

\begin{tabular}{llrl}
\hline Compound & Pre- $T_{\mathrm{m}} /{ }^{\circ} \mathrm{C}$ & $T_{\mathrm{m}} /{ }^{\circ} \mathrm{C}$ & $\begin{array}{l}\text { Temperature difference } \\
\text { between the two transitions } /{ }^{\circ} \mathrm{C}\end{array}$ \\
\hline DLPC & 34 & 91 & 57 \\
DMPC & 57 & 101 & 44 \\
DPPC & 70 & 106 & 36 \\
DSPC & 78 & 109 & 31 \\
\hline
\end{tabular}

without any re-crystallisation. This can be interpreted as evidence for the existence of heterogeneity in the morphology of the samples. From these observations, it can be confirmed that the PC samples undergo complex molecular rearrangement processes as a function of temperature. In addition, the acyl chain length dependence of these processes suggests that they are related to the hydrophobic regions of the multi-lamellar structures of the PCs studied.

An attempt was made to assess the temperature-dependent activation energy of both the pre-transition and the chain-melting transitions using conventional DSC.

(a)

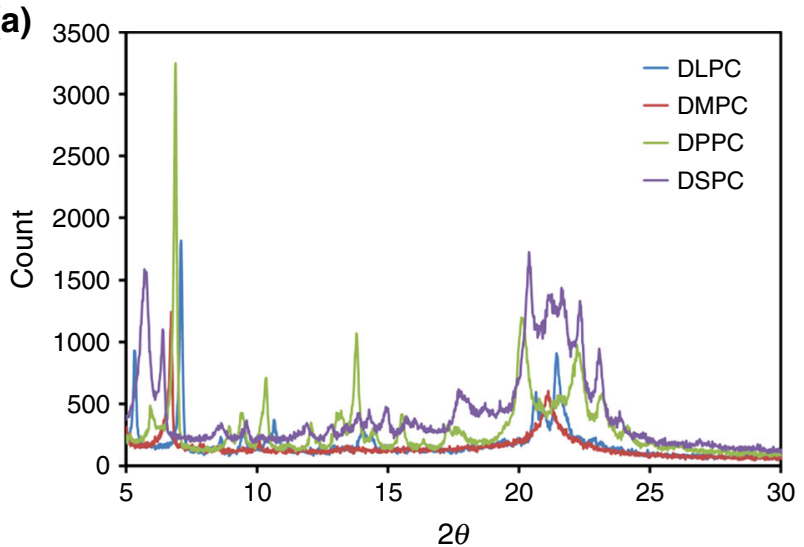

(b)

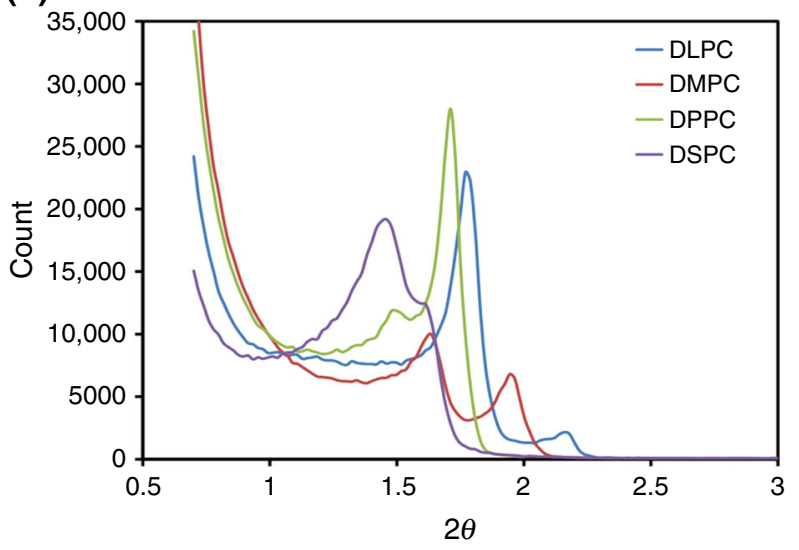

Fig. 2 XRPD results for the PCs after heating to $140{ }^{\circ} \mathrm{C}$ and cooling to ambient temperature (three times) for each sample. a Higher-angle diffractogram and $\mathbf{b}$ lower-angle diffractogram 

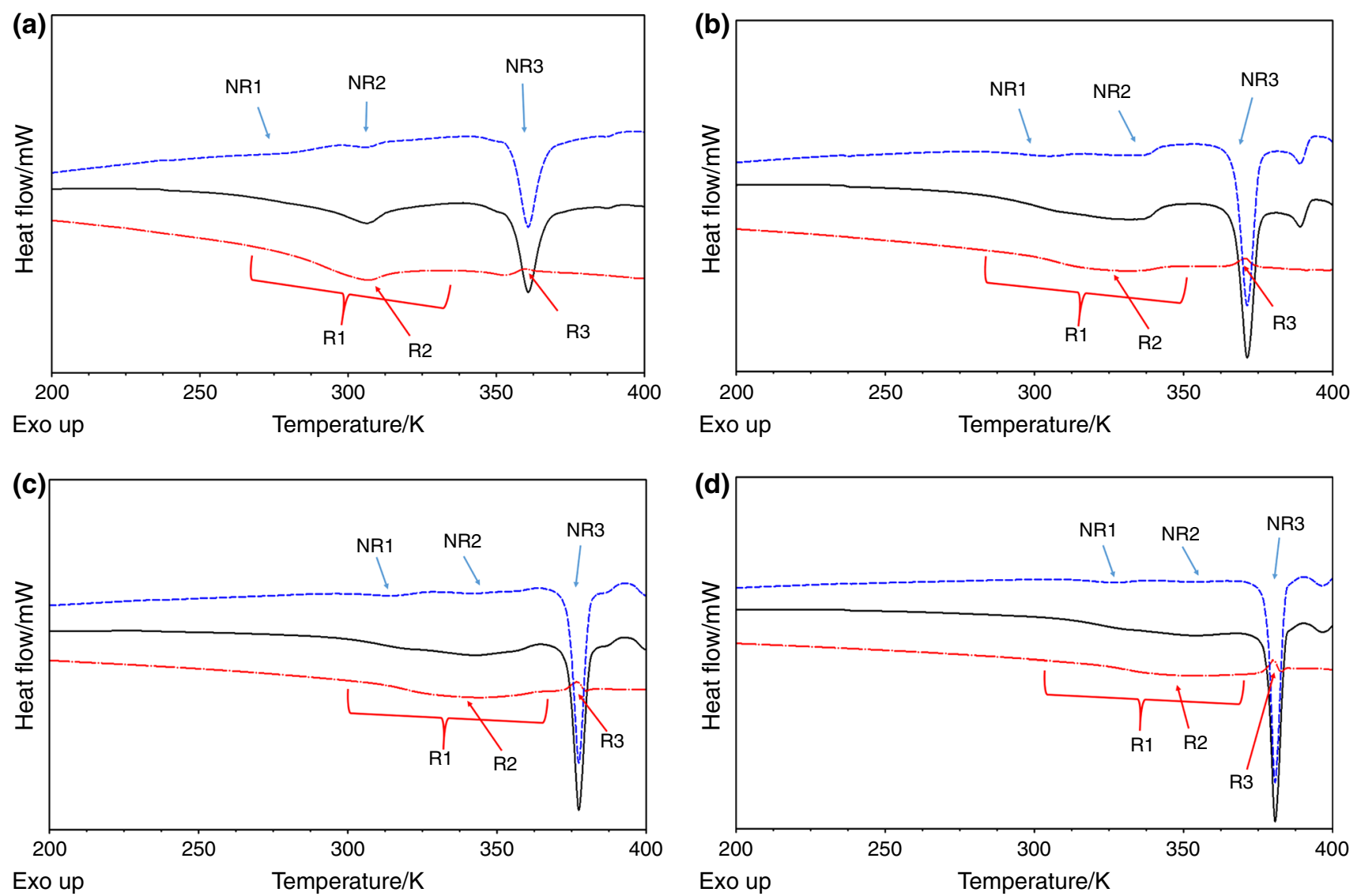

Fig. 3 Total, reversing and non-reversing TMDSC curves obtained for a DLPC, b DMPC, $\mathbf{c}$ DPPC and d DSPC. Data were obtained at an underlying heating rate of $2{ }^{\circ} \mathrm{C} \min ^{-1}$ and temperature amplitude of $1.5^{\circ} \mathrm{C}$ every $60 \mathrm{~s}$, using a sample size of $8.5 \pm 0.4 \mathrm{mg}$

However, the pre- $T_{\mathrm{m}}$ transitions did not show any temperature dependence, i.e. increasing the heating rate from 5 to $20{ }^{\circ} \mathrm{C} \mathrm{min}{ }^{-1}$ in increments of $5^{\circ} \mathrm{C} \mathrm{min}{ }^{-1}$ did not result in a significant shift in the transition temperature. For this reason, kinetic studies using DSC data could not be performed on the pre- $T_{\mathrm{m}}$ transitions. The chain-melting transition, on the other hand, exhibits significant temperature dependence, and thus kinetic studies were performed. It is also worth noting that the chain-melting transition is considered the most important transition for the functional properties of phospholipids in biological membranes and pharmaceutical liposomal formulations, as this transition changes bilayer integrity [18]. Hence it is of significant importance to understand the activation barrier that must be overcome for this process to proceed and how this varies with carbon chain length for phospholipids.

The apparent activation energies were calculated using Eq. (1), which have been previously described [19].

$\operatorname{In}\left(q \frac{\mathrm{d} \alpha}{\mathrm{d} T}\right)=\operatorname{In}(A f(\alpha))-\frac{E_{\mathrm{a}}}{R T}$

where $E_{\mathrm{a}}$ is activation energy, $A$ is the pre-exponential factor, $\alpha$ is the degree of conversion, $R$ is the universal gas constant, $q$ is heating rate, and $f$ is some mechanistic function of $(\alpha)$. The activation energy was obtained from the plot of the left-hand side of the equation versus $1 / T$ for a given degree of conversion $(\alpha)$ at the different heating rates employed. The $R^{2}$ values of the Arrhenius plots were $\geq 0.95$. The dependence of the apparent activation energies on the degree of conversion $(\alpha)$ for the phosphatidylcholines is presented in Fig. 4.

The data acquired show the existence of a strong dependence of the apparent activation energy on the degree of conversion $(\alpha)$ for chain-melting transition of all four phosphatidylcholines studied. In all four samples, the activation energy decreased as the reaction progressed (Fig. 4; Table 3). The observed behaviour in apparent activation energy could explain/support the cooperative nature of the chain-melting transitions. In this case, relatively high apparent activation energy is required initially for the transition to begin, i.e. at the initial stage, a high degree of cooperation is required for molecules to move about their local environments; hence, greater energy is required. As molecules gain energy, the volume of the sample increases leading to increase in intermolecular distances and thus reducing the degree of intermolecular interactions. 


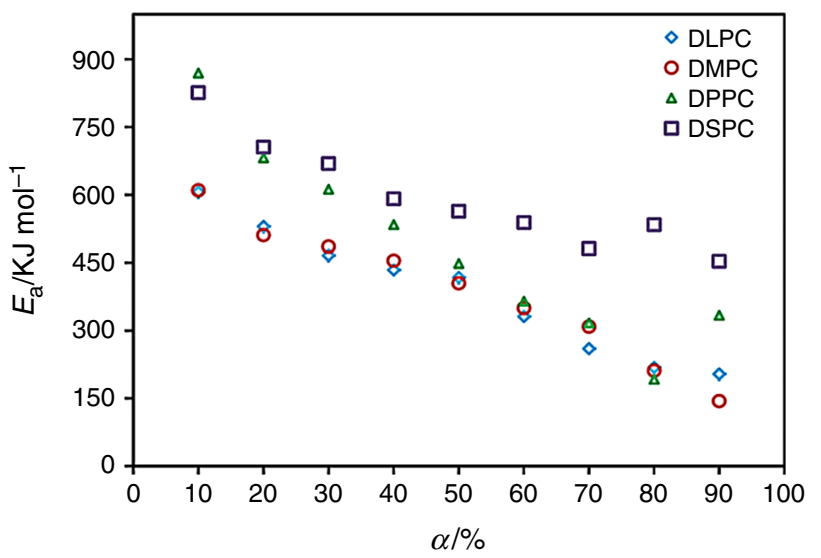

Fig. 4 Plots of activation energy $\left(E_{\mathrm{a}}\right)$ versus the degree of conversion $(\alpha)$ for the chain-melting transition $\left(T_{\mathrm{m}}\right)$ of DLPC (open blue diamond), DMPC (open brown circle), DPPC (open green triangle) and DSPC (open purple square). (Color figure online)

Table 3 DSC data of calculated mean apparent activation energies of the chain-melting transition at each value of $\alpha$ for DLP, DMPC, DPPC and DSPC $(n=3)$

\begin{tabular}{lllll}
\hline$\alpha / \%$ & $E_{\mathrm{a}} / \mathrm{kJ} \mathrm{mol}^{-1}$ & & & \\
\cline { 2 - 5 } & DLPC (12:0) & DMPC (14:0) & DPPC (16:0) & DSPC (16:0) \\
\hline 10 & $606 \pm 50$ & $610 \pm 24$ & $869 \pm 90$ & $826 \pm 45$ \\
20 & $530 \pm 43$ & $511 \pm 45$ & $682 \pm 31$ & $705 \pm 38$ \\
30 & $466 \pm 51$ & $486 \pm 19$ & $612 \pm 28$ & $669 \pm 55$ \\
40 & $434 \pm 23$ & $455 \pm 18$ & $534 \pm 42$ & $591 \pm 31$ \\
50 & $418 \pm 34$ & $405 \pm 36$ & $448 \pm 20$ & $563 \pm 62$ \\
60 & $331 \pm 18$ & $350 \pm 20$ & $365 \pm 29$ & $538 \pm 44$ \\
70 & $260 \pm 21$ & $309 \pm 27$ & $317 \pm 17$ & $481 \pm 26$ \\
80 & $218 \pm 24$ & $211 \pm 18$ & $192 \pm 10$ & $534 \pm 43$ \\
90 & $204 \pm 22$ & $144 \pm 8$ & $334 \pm 27$ & $453 \pm 25$
\end{tabular}

Comparison of the apparent activation energies obtained for the PC homologues show, in general, that the energy required for the chain-melting transition increased with increasing carbon chain length (Table 3 ). This is only true if DLPC is ignored and can, again, be attributed to the ability of phospholipids with longer diacyl chain lengths to undergo greater hydrophobic interactions.

\section{Conclusions}

The results presented herein have shown the existence of new transitions prior to the isotropic melt for dehydrated saturated phosphatidylcholines. This study also reports the existence of a pre- $T_{\mathrm{m}}$ transition for dehydrated DLPC, DMPC, DPPC and DSPC (at $34 \pm 1,57 \pm 1,70 \pm 1$, $78 \pm 1{ }^{\circ} \mathrm{C}$, respectively), which may have significant implication on application of the materials in the solid state, particularly in freeze-dried liposomal drug delivery systems.

Temperature-modulated DSC revealed that the broad pre- $T_{\mathrm{m}}$ transition is composed of energetically weak, complex overlapping processes. It was found that DLPC, DMPC, DPPC and DSPC undergo two phase transitions in the non-reversing heat flow signal related to heterogeneity in sample morphology when analysed by MTDSC. The main process that was identified, the $T_{\mathrm{m}}$, is complex and together with the endothermic peak in the non-reversing heat flow gives a small exothermic signal in the reversing heat flow, which is attributed to the small recrystallisation followed by partial melting of PC lamellae. The peak temperature at which the overall pre- $T_{\mathrm{m}}$ and $T_{\mathrm{m}}$ processes occur is found to be diacyl carbon chain length dependent, i.e. increasing diacyl chain length increases the peak temperature of the transition. Kinetic studies of the chainmelting process show the activation energy of this process to decrease as the transition progresses. This behaviour has been attributed to the decrease in the degree of intra- and inter-molecular cooperation required for molecular orientations to occur.

The information provided in this study and such studies of phospholipid systems can greatly enhance understanding how to best optimise the application of phospholipids in pharmaceutical delivery systems and other technologies.

Acknowledgements This study was funded by the University of Greenwich. Samuel K Owusu-Ware wishes to thank the University of Greenwich for a bursary in order to conduct his doctoral studies.

\section{Compliance with ethical standards}

Conflict of interest The authors declare no competing financial interest.

Open Access This article is distributed under the terms of the Creative Commons Attribution 4.0 International License (http://crea tivecommons.org/licenses/by/4.0/), which permits unrestricted use, distribution, and reproduction in any medium, provided you give appropriate credit to the original author(s) and the source, provide a link to the Creative Commons license, and indicate if changes were made.

\section{References}

1. Ulrich AS. Biophysical aspects of using liposomes as delivery vehicles. Biosci Rep. 2002;22(2):129-50.

2. Stark B, Pabst G, Prassl R. Long-term stability of sterically stabilized liposomes by freezing and freeze-drying: effects of cryoprotectants on structure. Eur J Pharm Sci. 2010;41:546-55.

3. Popova AV, Hincha DK. Thermotropic phase behavior and headgroup interactions of the nonbilayer lipids phosphatidylethanolamine and monogalactosyldiacylglycerol in the dry state. BMC Biophys. 2011;4:11.

4. Koynova R, Caffrey M. Phases and phase transitions of the phosphatidylcholines. Biochim Biophys Acta. 1998;1376:91-145. 
5. Popova AV, Hincha DK. Intermolecular interactions in dry and rehydrated pure and mixed bilayers of phosphatidylcholine and digalactosyldiacylglycerol: a fourier transform infrared study. Biophys J. 2003;85:1682-90.

6. Popova AV, Hincha DK. Effect of cholesterol on dry bilayers: interactions between phosphatidylcholine unsaturation and glycolipid or free sugar. Biophys J. 2007;93:1204-14.

7. Chapman D, Byrne P, Shiply GG. The physical properties of phospholipids. I. Solid state and mesomorphic properties of some 2,3-diacyl-DL-phosphatidylethanolamines. Proc R Soc A. 1966;290(1420):115-42.

8. Chapman D, William RM, Ladbrooke BD. Physical studies of phospholipids. VI. Thermotropic and lyotropic mesomorphism of some 1,2-diacyl-phosphatidylcholines (Licithins). Chem Phys Lipids. 1967;1(5):445-75.

9. Fringeli UP. A new crystalline phase of 1- $\alpha$-dipalmitoyl phosphatidylcholine monohydrate. Biophys J. 1981;34:173-87.

10. Crowe JH, Hoekstra FA, Nguyen KHN, Crowe LM. Is vitrification involved in depression of the phase transition temperature in dry phospholipids. Biochim Biophys Acta. 1996;1280:187-96.

11. Chapman D. Liquid crystals and cell membranes. Ann N Y Acad Sci. 1966;137(2):745-54.

12. Wang B, Tan F. DSC study of phase transition of anhydrous phospholipid DHPC and influence of water content. Sci China B. 1997;40(4):412-8.
13. Marsh D. General features of phospholipid phase transitions. Chem Phys Lipids. 1991;57:109-20.

14. Sauer BB, Kampert WG, Blanchard EN, Threefoot SA, Hsiao BS. Temperature modulated DSC studies of melting and recrystallization in polymers exhibiting multiple endotherms. Polymer. 2000;41:1099-108.

15. Sauer BB, Kampert WG, McLean RS. Thermal and morphological properties of main chain liquid crystalline polymers. Polymer. 2003;44:2721-38.

16. Qui Z, Komura T, Nishi T. DSC and TMDSC study of melting behaviour of poly(butylene succinate) and poly(ethylene succinate). Polymer. 2003;44:7781-5.

17. El-Taweel SH, Hohne GWH, Mansour AA, Stoll B, Seliger H. Glass transition and the rigid amorphous phase in semicrystalline blends of bacterial polyhydroxybutyrate (PHB) with low molecular mass atactic R,S-PHB-diol. Polymer. 2004;45:983-92.

18. Leekumjorn S, Sum AK. Molecular studies of the gel to liquidcrystalline phase transition for fully hydrated DPPC and DPPE bilayers. Biochim Biophys Acta. 2007;1768:354-65.

19. Keuleers RR, Janssens JF, Desseyn HO. Comparison of some methods for activation energy determination of thermal decomposition reactions by thermogravimetry. Thermochim Acta. 2002;385(1-2):127-42. 